\title{
A COMPANION TO THE PHILOSOPHY OF TIME
}

Edited by Adrian Bardon and Heather Dyke

\author{
Time Travel and Time Machines \\ Douglas Kutach
}

\begin{abstract}
Thinking about time travel is an entertaining way to explore how to understand time and its location in the broad conceptual landscape that includes causation, fate, action, possibility, experience, and reality. It is uncontroversial that time travel towards the future exists, and time travel to the past is generally recognized as permitted by Einstein's general theory of relativity, though no one knows yet whether nature truly allows it. Coherent time travel stories have added flair to traditional debates over the metaphysical status of the past, the reality of temporal passage, and the existence of free will. Moreover, plausible models of time travel and time machines can be used to investigate the subtle relation between space-time structure and causality.
\end{abstract}

Time travel is a relatively recent subject of philosophical inquiry, having gained little discernible intellectual traction before the twentieth century. Since then, interest in the implications of time travel has grown significantly, largely as a result of the empirical success of Einstein's general theory of relativity, which provides for the physical possibility of time travel to the past, effectively appropriating the subject of time travel from the realm of pure fiction for use in scientific inquiry. Physically plausible models of time travel are now an important topic of consideration for anyone investigating the nature of time.

Much of the philosophical literature on time travel attempts to engage with traditional philosophical problems of free will, personal identity, and the passage of time. The extent to which time travel brings genuinely new resources to bear on these topics is debatable, but it is fair to say that time travel scenarios can at least help liberate one's mind from the grip of naive intuitions and guide one towards a superior conceptualization of the connections among action, causation, time, change, and related notions. For example, many people believe in some form of fatalism about the past, holding that the past cannot be influenced. Physically plausible models of time travel can call attention to the possibility that the kind of causal interactions we are familiar with in our everyday lives is compatible with our being able to influence the past. That in turn can raise awareness of the possibility that we exert influence over the past in mundane circumstances without traveling back in time.

\section{Defining Time Travel}

A basic grasp of what constitutes time travel can be gleaned from stock examples in science fiction. Paradigmatically, time travel consists of a person at some initial time $t_{i}$ commencing a journey that lasts for a duration $\Delta t$ and completing the journey at some final time $t_{f}$ that is not equal to $t_{i}+\Delta t$. Traveling to the future paradigmatically involves $t_{f}$ occurring substantially after $t_{i}+\Delta t$ and traveling to the past involves $t_{f}$ occurring substantially before $t_{i}$. Using the terminology of David Lewis (1976), we can refer to the $\Delta t$ as the duration of the time traveler's personal time and the $t_{f}-t_{i}$ as the duration of external time, the time through which the time traveler travels. This preliminary characterization of time travel as a mismatch between personal time and external time can be made more precise along several dimensions.

First, one can specify the concept of personal time more carefully by characterizing what it means for a person to experience a certain duration of time. Paradigmatically, a time traveler to the future rides a time machine for twenty seconds or so and exits to find that hundreds of years have passed for everything else. The personal time is recorded in the memory and other bodily 
changes of the time traveler as well in her pocket watch. External time is recorded on all the other clocks and calendars in the world. In clarifying what personal time measures, one might choose to disregard the pocket watch altogether and associate personal time merely with how long the journey seems to last for the traveler. One could count cryonics-the freezing and restoration of a person-as a form of time travel to the future because a recently thawed woman can rightly judge that "shortly" after being told that the freezing will begin, she wakes up thousands of years in the future. In principle, the conditions required for time travel could be so liberally interpreted that a mere nap would count as time travel, but 'time travel' does not ordinarily include these routine mismatches between the durations measured by a mechanical clock and by a person's internal clock. A more restrictive construal of personal time could require that it track the rate at which fundamental processes take place. For example, if there were some region of the universe where all physical processes slow down relative to other locations, passing through that region would count as genuine time travel to the future. Interestingly, because of the effects described by relativity theory, time travel according to this stricter definition does actually take place. The theory of relativity thus helps to mitigate concern about the vagueness inherent in the concept of personal time because it grounds a sense of temporal duration in which all local physical processes can take place at a rate that differs from their surrounding environment. A person who travels on a round trip to a distant star will return having experienced less time than people who stay on Earth and thus will have traveled into the future. Because this form of time travel is based on well-confirmed physics, it is uncontroversial that time travel towards the future exists.

The idea of visiting the past raises more probing questions about what it means to travel through time. If there were some magical process by which a wizard could wave a wand and thereby make a person vanish from the present and appear a century ago, that would seem to count as a form of time travel. However, it is difficult to establish clear criteria in full generality for what kind of connection is needed among the various temporal stages of a potential time traveler in order for her to count as a bona fide time traveler to the past. Presumably, the criteria used in making such judgments draw on resources familiar from debates over the nature of personal identity-for example, having a sufficient degree of mental continuity and the appropriate sort of causal linkage among the various stages of a person's life. Judgments can be murky, though, in part because some time travel scenarios are subject to a reinterpretation that eschews time travel altogether. For example, the classic time machine described by H. G. Wells (1895), requires the spatio-temporal path of the putative time traveler to form a zigzag shape where the journey starts on a future-directed physical evolution, backtracks towards the past until the destination is reached and then backtracks again towards the future when the passenger exits the time machine. If the fundamental laws of nature are akin to those posited in paradigmatic theories of fundamental physics, such Wellsian time travel can be reinterpreted as an entirely future-directed process: the spontaneous generation of a person in a time travel machine, who immediately fissions into a rather ordinary future-directed human stepping out of the machine together with a copy of that same human who evolves in slow motion reverse towards the future inside the time machine, becoming ever so slightly younger hour by hour until the machine collides with a virtually identically structured machine with a virtually identical human, resulting in the merging of all their parts followed immediately by their complete vanishing. This possible reinterpretation should not by itself prevent such a scenario from counting as genuine time travel because it does not rule out the existence of a reliable method by which one could bring about the temporal zigzagging at will from the initial stage when the time traveler activates the time machine. That said, there are solid reasons (Kutach 2011) for thinking Wellsian time machines cannot be manufactured in our universe. 
Finally, one of the trickier aspects of defining time travel is to distinguish it from cases of travel to an alternate world. This issue arises especially in attempted models of time travel where some past $P$ occurs, but then someone travels into the past and prevents $P$, thereby requiring that there be one sense in which $P$ happened and another sense in which it did not happen. This possibility could be modeled in terms of multi-valued physical fields, but the mere existence of multi-valued fields does not by itself constitute the kind of alteration of the past countenanced in science fiction because the multiple values of the field might have equal status as existents. Some further structure is required to justify a sense in which $P$ happened "first" and then was "later" prevented rather than history merely evolving such that one magnitude of the multivalued field instantiated $P$ and the other didn't. One can attempt to cash out the idea that history can be altered in terms of a multiverse model of the universe or with a two-dimensional model of time (Meiland 1974), but a recurring worry about such schemes is that they do not vindicate the claim that the time traveler has traveled into the actual past. Instead, such models are often better described as instantiating travel to an alternate world that is qualitatively similar to the time traveler's actual past.

\section{Temporal Passage}

The possibility of past-directed time travel has been employed in debates over the metaphysics of temporal passage. Some of this literature attempts to accommodate time travel in presentist conceptions of time, where only the present is real. (See [PRESENTISM, ETERNALISM, AND GROWING BLOCK THEORY].) The primary concern is that presentism appears to be incompatible with the existence of past-directed time travel. Because humans have no evidence of any actual time travel to the past, it poses only a hypothetical threat to presentism, but one could investigate whether it is compatible with time travel to the past anyway. The reason presentism appears to disallow time travel to the past is that the presentist denies the existence of the past, and one cannot travel to a non-existent location. Keller and Nelson (2002) and Monton (2003) defend the compatibility of presentism and past-directed time travel by arguing in effect that presentists already need to account for how the actual future and actual past differ from non-actual futures and non-actual pasts, so one can simply leverage one's favorite presentist account of the actual past to underwrite truths about time travel. Sider (2005) questions the success of this strategy by countering that genuine time travel requires personal time to be the same kind of time that ordinary people experience when not time traveling. For the presentist, the normal passage of time essentially incorporates the presentist's fundamental flow of time, but the personal time of the time traveler must be characterized without recourse to fundamental temporal passage. This discrepancy appears to disallow any hypothesized pastdirected physical processes from counting as bona fide personal time and thus from counting as genuine time travel.

Another subset of the philosophical literature attempts to use the physical possibility of pastdirected time travel to attack dynamic conceptions of time. The most famous such argument is Gödel's (1949) classic attack on the reality of time. According to Gödel, the physical possibility of space-time structure with temporal loops-rendered technically as closed time-like curvesmotivates the conclusion that time is "ideal" in the sense of not having an "objective lapse of time," thus vindicating Parmenides and Kant, who deny "the objectivity of change and consider change as an illusion." Gödel's paper is brief and underspecifies the key concepts, so readers will benefit from recent commentaries. A sympathetic reading of Gödel's argument can be found in Horwich (1993), while an antagonistic reading can be found in Earman (1995).

One take on Gödel's argument-aimed more at fashioning his core idea into a passable argument than at presenting an accurate rendition of his writings-is that Gödel is groping towards the conclusion that the passage of time is not part of fundamental reality but exists 
derivatively, similar perhaps to how the solidity of a granite block is a cognitive simplification. (See Savitt (1994) and Yourgrau (1991) for related readings.) Gödel presupposes a conception of fundamental temporal passage involving what he calls an "objective lapse of time" by which he apparently means an exhaustive foliation (or slicing) of space-time into a linearly ordered set of metaphysically distinguished global time slices. The non-existence of fundamental temporal passage is defended on epistemological grounds: Even if the actual world contains an arrangement of matter sufficient for a preferred foliation of space-time into global time slices, there are other physically possible worlds with observers who evolve locally just as we do-with the same laws and same kinds of matter-but which contain no global foliation. The hypothetical inhabitants of these Gödelian worlds would report all the same observations about the passage of time that we do because locally they behave just like us. Yet, because there is no "objective lapse of time" in such worlds owing to the lack of any global foliation, they cannot be responding to an objective lapse of time. Because we evolve through time exactly like they do, it is plausible that we are not detecting or responding to an "objective lapse of time" even if there is one. Thus, our experience of temporal passage provides no evidence for the existence of fundamental temporal passage.

In the end, this Gödel-inspired argument appears ineffective against fundamental temporal passage per se, largely because one could model fundamental temporal passage without requiring any foliation of space-time. More tellingly, the strength of the above argument derives not from anything specifically related to time travel but primarily from its assumption that the motion of matter (and thus presumably our awareness) is entirely unresponsive to the presence or absence of the hypothesized fundamental passage. The overall argument might be summarized as follows: Given that matter evolves according to laws that can be construed as operating regardless of whether fundamental temporal passage exists, temporal passage can be safely dropped from one's theory of fundamental reality without hindering one's ability to explain all the empirical phenomena that motivate our talk of temporal passage. This argument holds just as well for ordinary space-times without time travel.

\section{Indeterminism and Consistency Constraints}

Time travel in general relativity is understood to take place through temporal loops, which are formalized as closed time-like curves (CTCs). The presence of CTCs in an otherwise unassuming space-time can have two opposing effects. On the one hand, they can prevent events from determining what happens in the future, and on the other hand, they can impose severe and unfamiliar restrictions on how matter behaves. For illustration, we can consider a twodimensional space-time where two space-like line segments are topologically identified, as shown in Fig. 18.1, to constitute a simplistic wormhole. In this toy model, it is helpful to ignore the physical evolution of matter leading up to the surface $\Sigma_{1}$ and leading away from $\Sigma_{2}$ and just concentrate on the time travel that occurs when particles or fields strike the underside of $\Sigma_{2}$ and pop out automatically in the past on the top side of $\Sigma_{1}$.

The wormhole depicted in Fig. 18.1 highlights an important kind of indeterminacy that can occur in virtue of CTCs. A state of affairs (before $\Sigma 2$ exists) with a single particle passing from left to right could lawfully evolve either into a situation where the particle goes straight through the gap between $\Sigma 1$ and $\Sigma 2$, or it could evolve so as to ricochet off its future self into the wormhole, whence it ricochets off its past self and continues its travel to the right. Because nothing in the state prior to $\Sigma_{2}$ suffices to settle whether the particle collides with itself, determinism cannot hold true of this model. Furthermore, the lack of determination in the neighborhood of the wormhole is not constrained by any probabilistic principle. The wormhole itself certainly does not fix the relative likelihood of the two scenarios in Fig 18.1. Thus, we seem 
to have a physically possible circumstance where the physical evolution is indeterministic, yet neither chancy nor wholly unconstrained by laws.

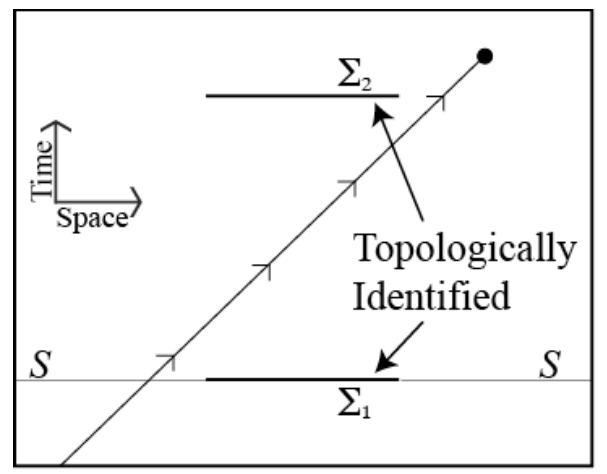

Particle Passes Through Freely

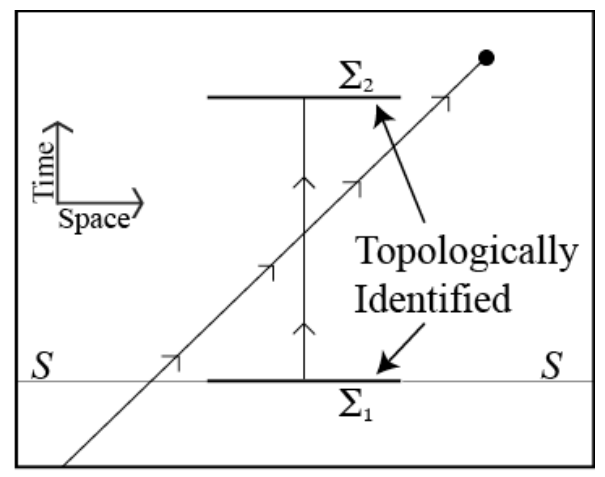

Particle Collides with Itself

\section{Figure 18.1}

This same wormhole also illustrates how space-times with CTCs can impose severe constraints on the behavior of matter. Any evolution of the physical state at $\Sigma_{1}$ (in conjunction with everything else, $S$, happening at that time) must be such that it evolves into that very same state again when it reaches $\Sigma_{2}$ because $\Sigma_{1}$ and $\Sigma_{2}$ are two labels for the same location.

Worries about whether the history of a time traveler will unfold consistently are often presented in terms of the infamous grandfather paradox, the traditional story where a time traveler attempts to prevent his own birth. Any scenario where a person or device is set to countermand what has happened in the past and thereby create a contradictory state of affairs is known as bilking. Consistent explications of attempts to bilk the past are routinely structured so that regardless of how many repeated voyages are made to the past, events always work themselves out in a unique contradiction-free manner. After all, any actual attempt to travel back in time to prevent one's own birth must fail once we take for granted that the time traveler was born and grew up in the usual manner. However, it may still appear paradoxical to claim that the time traveler is guaranteed not to prevent his own birth while at the same time holding that the time traveler interacts with his environment just like other inhabitants and thus has the ability to help grandpa find an early end.

The canonical answer to the grandfather paradox is laid out in Lewis (1976) where it is pointed out that the paradox trades on an equivocation between two different notions of possibility. The key idea can be summarized in two parts. First, the local fragment of the world that instantiates the time traveler's attempted attack on his grandfather is such that the time traveler is the same as non-time-travelers with regard to causation, influence, action, and related notions. For example, in the scenario the potential assassin actually misses his target or loses his nerve, but hypothetically altering him to aim more accurately or to strengthen his resolve would by physical law very likely lead to grandfather's quick demise. Second, there is no global state of affairs that both retains the continuous existence of the time traveler (as a normal human born in the usual way) around the temporal loop as well as the successful prevention of his birth. So it is locally possible for the time traveler to prevent his future birth, but it is not globally possible because that would be self-contradictory.

What appears to be a signature effect of CTCs is that some physical states that are possible according to the usual laws governing allowable local states cannot be extended via the usual laws of temporal evolution into a globally consistent solution. A restriction on the space of all 
physically possible fragments of history to those that can be extended by law into a globally consistent solution of a maximally large space-time is called a consistency constraint. Consistency constraints reveal themselves not only in cases of bilking but also in scenarios where a macroscopic object exists only along a CTC. For example, a book that is never manufactured but is passed along from a time traveler to her younger self will need to evolve around a CTC so that any weathering of the pages will vanish somehow when it persists all the way around its circular history.

A philosophical debate has developed which attempts to adjudicate the status of these consistency constraints, in particular how they relate to physical and logical possibility. Stephen Hawking (1992) conjectures that the laws of physics forbid all time travel to the past in order to avoid the threat of the grandfather paradox. He invokes what he calls the "chronology protection conjecture,' that "the laws of physics prevent closed time-like curves from appearing." (p. 604) The underlying motivation for exploring the chronology protection conjecture appears to be the thought that laws are needed to make "the universe safe for historians." (p. 603) Alternatively, one could argue that chronology protection is otiose because logic alone already ensures that whatever actions a time traveler takes in the past will fail to contradict what actually happened. There is no need for a law of nature to rule out paradoxical possibilities because it is already logically impossible for the actual world to instantiate contradictory states of affairs.

Arntzenius and Maudlin (2002) label this appeal to logical consistency the "stonewalling response" to worries about consistency constraints, summarized as follows:

In order to satisfy such constraints one needs some pre-established divine harmony between the global (time travel) structure of space-time and the distribution of particles and fields on spacelike surfaces in it. But it is not plausible that the actual world, or any world even remotely like ours, is constructed with divine harmony as part of the plan. In fact, one might argue, we have empirical evidence that conditions in any spatial region can vary quite arbitrarily. So we have evidence that such constraints, whatever they are, do not in fact exist in our world. So we have evidence that there are no closed time-like lines in our world or one remotely like it.

As Dowe (2007) and others have pointed out, consistency constraints do not need to be enforced by laws of nature. There are two kinds of regions that need to be addressed separately. The first are locations within a causal loop, where CTCs are short enough in duration to require that matter behaves in unusual ways in order for the stuff in the causal loop to evolve consistently. We do have zero evidence of matter actually behaving in such unusual ways, and that does license us to infer that we are not a part of a tight causal loop. (We might still be part of a causal loop so large that the imposed constraints are difficult to discern.) But no one in the debate over the status of consistency constraints is arguing that there are time travelers from the future in our midst, so that conclusion is old news.

The second kind of location lies outside of all causal loops, especially states that precede a CTC like the $S$ in Fig. 18.1 that instantiates everything happening outside $\Sigma$ 1. Depending on the laws, there might be severe consistency constraints on states like $S$, but no one has provided any reason to think these constraints on $S$ are likely to reveal themselves well ahead of time by imposing unfamiliar restrictions on our ability to arrange macroscopic objects seemingly at will. Thus, we are in a very poor position to conclude that CTCs are absent from our future.

As to the claim that worlds with constraints are not remotely like our world, one could respond that such worlds would be like our world with respect to all its fundamental laws and all the material occupying space-time and so would be very nearly like our world in most fundamental respects. It is true that the consistency constraints imposed by CTCs are unlike other kinds of 
constraints we have empirically observed, but the above arguments provide a good explanation for why we haven't observed consistency constraints even if future generations invent time machines.

The consistency constraints can be made to appear more objectionable than they really are by framing them with standard plot devices from fiction (Smeenk and Wüttrich 2011). The assassin who makes numerous journeys into the past to prevent his own birth may first fail to bilk the past by poisoning the wrong victim, then fail on the second trip by slipping on a banana peel and misfiring his musket, then fail on the third trip by being kidnapped by pirates before the crucial showdown, and so on. Such happenings would indeed be strikingly improbable, but these solutions are implausible predictions for what will happen if there is a CTC and someone attempts to enter it with the intention of bilking the past. What is dodgy about these storybook failures is that they all instantiate developments where things happen fairly normally except perhaps for a brief moment where an improbable mishap occurs. A more plausible hypothesis is that the entire causal loop would behave so as to deviate minimally radically throughout the CTC while still securing overall consistency. Rather than evolving ordinarily for most of the CTC with an extremely radical departure for a brief period, there would be a continual amalgamation of less severe departures from the prototypical behavior of macroscopic objects. This hypothesis cannot be tested without observing an actual CTC, but if it is true, the resulting causal loop would not be strikingly improbable given that consistency constraints must be obeyed.

\section{Time Machines}

When nature provides a suitable space-time, a time traveler need only travel along a CTC in order to arrive in the past, but if CTCs are not lying around in the environment, is there anything an aspiring time traveler can do to create a CTC? This is the question, "Are time machines physically possible?" In the academic literature, a time machine is intended to be a local physical condition (like pushing a button or pulling a lever) such that its occurrence causes (or produces or brings about) a CTC. A time machine does not need to be a mechanical contraption that a time traveler rides when circumnavigating a CTC, and the requirement that the activation of the time machine be local is often interpreted liberally.

Although the question of whether it is possible to build a time machines is interesting to the general public, readers who delve into the academic literature will immediately encounter more than a few technicalities and an abundance of esoteric models of general relativity. That is because the question about the physical possibility of time machines (beyond that of time travel in general) is useful for exploring constraints on space-time structure, including how to handle the possibility of singularities, which are edges or holes or pathological infinities in the structure of space-time itself. Of special interest is cosmic censorship, the hypothesis that no space-time singularities display the kind of unrestricted indeterminacy illustrated in Fig. 18.1. Cosmic censorship is relevant to the possibility of time machines because it is understood to imply chronology protection. Earman, Wüttrich and Smeenk (2009) provide an excellent entry point into these issues for readers with some basic knowledge of general relativity.

One surprising feature of the technical literature is that its conception of a time machine is significantly at odds with most science fiction where prototypically a person enters a time machine, activates some buttons or levers, and shortly thereafter arrives in the past. The time traveler's activation of the machine is presumed to have caused the time traveling to occur and thus (when the discussion is framed in terms of general relativity) to have caused a CTC that includes the activation event as part of its temporal loop. This kind of time machine is typically ruled out or set aside in the technical literature. Instead, a time machine is conceived as a localized event that creates a CTC lying entirely in that event's future. The reason cited by 
Earman (1995, p. 189) and (Earman, Wüttrich and Smeenk 2009) is that the goal is to characterize a time machine in a way that captures the idea that the activation of the time machine produces the CTC. Because the successful operation of a prototypical time machine would by definition have a CTC existing just before the time machine is activated, the worry is that the alleged time machine would not count as having produced a CTC that "already" exists.

This reasoning can be faulted for incorporating too much of the folk conception of causal asymmetry into what counts as the relevant sort of causation. It is especially surprising to define time machines so that they cannot be used to visit the past because-given that the physical possibility of CTCs has already been accepted-one presumably should not discount the possibility of an event influencing its immediate past.

There is a way to define a time machine that satisfies all the necessary conditions for a time machine without ruling out prototypical scenarios of time travel to the past. For a sketch of how this works, let us first define an event to be a nomologically possible region of space-time augmented with a complete specification of all the fundamental fields and particles inhabiting that region. For illustration, it is convenient to restrict consideration to a region that is a connected subset of a single space-like hyper-surface so that any two points in that region can be connected to each other along an everywhere space-like curve. Consider an event $e$ that includes a part $c$ that is suitably small and instantiates a human pressing a button marked 'Go.' Let $b$ be everything else in $e$. The localized event $c$ and its background $b$ together constitute the entire event $e$. We can also consider an alternative event $e^{*}$ which is just like $e$ except that $c$ is altered to form $c^{*}$, an event where the human does not press the button.

The fundamental laws of nature might be such that when they are applied to $e$ in order to fill out what happens elsewhere in virtue of $e$ (going both forward and backward in time if the laws allow), there is a consistent result in the form of a larger fragment of space-time that includes CTCs going through $c$. It also might be that when the fundamental laws are applied to $e^{*}$ to draw out its consequences for what happens elsewhere, the resulting filled-out space-time region includes no CTCs. It is fair to say of such cases (where $e$ happens and there are CTCs) that the occurrence of $e$ rather than $e^{*}$ is responsible for the appearance of CTCs. Because $e$ and $e^{*}$ differ only in what happens in the local region of $c$, it is also fair to say that (in background conditions $b$ ) the occurrence of $c$ rather than $c^{*}$ is responsible for the appearance of CTCs. We can now state a preliminary definition that applies when the fundamental laws are deterministic in character: A time machine is physically possible just in case there are physically possible background conditions $b$ such that there are two ways of filling in a localized foreground region, one of which determines that a CTC exists and the other of which determines that no CTCs exist.

A more thorough discussion would extend it to handle chancy laws and temporally extended events, but these complications can be incorporated relatively easily. In any case, it should be clear enough that this scheme allows us to make sense of how local conditions can make a causal difference as to whether a CTC exists without requiring any metaphysical resources other than the (already presumed) fundamental laws. Whether time machines exist according to this definition is not currently known. An adequate answer presumably requires further clarification of how topological principles are related to fundamental laws because even if some $e$ could be proven to evolve towards the future and towards the past in a way that instantiates two perfectly matching wormhole interiors, the laws governing the temporal evolution of matter would not by themselves ensure that the two interior edges would connect.

Returning to the conventional conception of time machines as localized events that produce CTCs lying entirely towards the future, a major philosophical problem-summarized well by 
Earman, Smeenk, and Wüttrich (2009)-is that it is difficult to specify a suitably precise technical definition for a time machine. The primary problem is that the possibility of a CTC in the future goes along with a lack of determination relations holding between what happens previous to the potential appearance of the CTC and what happens after the potential appearance of the CTC. This lack of determination is similar to the indeterminacy introduced by the wormhole of Fig. 1 because neither is subsumed under a probabilistic rule. However, it is not exactly the same because the failure here leaves open what the future spatio-temporal structure is (and perhaps whether there is a CTC at all) rather than merely leaving open how matter evolves around the given CTC. As a result, attempts to specify a time machine in terms of a localized state that determines the existence of a later CTC needs to be carefully massaged to capture the idea that time machines ensure that all appropriate spatio-temporal extensions of the time machine's region instantiate a CTC.

Even without clarifying a sufficient condition for a time machine, one could address the project of showing that plausible physical constraints make a time machine impossible, for example that energy densities are never negative. This project has generated some attention because theorems (Hawking 1992, Krasnikov 2003) have attempted to demonstrate that under certain (arguably plausible) physical assumptions, time machines cannot exist, although Krasnikov's no-go theorem in particular has been called into doubt (Manchak 2011). Needless to say, there is plenty of technical and conceptual work to be done in order to figure out the best ways to define a time machine and assess their possibility. Because the details are too technical to take up here, interested readers are advised to consult Earman, Smeenk, and Wüttrich (2009) or Earman and Wüttrich (2010) to begin following up on such matters.

\section{Quantum Mechanics}

Quantum mechanics bears on several aspects of time travel. For one brief technical example, it has been argued (Thorne 1994) that quantum mechanical models can justify the existence of the kind of exotic material needed to keep a wormhole open long enough for a time traveler to pass safely through.

In a more probative application, quantum mechanics can call into question the legitimacy of relying on general relativity to provide a decent grasp of physical possibility. It is famously difficult to formulate a coherent theoretical framework that meshes quantum mechanics with general relativity, and it may turn out on further examination that the CTCs permitted by general relativity are an artifact of having too broad a conception of physical possibility. Perhaps in order to achieve peaceful coexistence between general relativity and quantum mechanics, one needs an alternative interpretation of general relativity with no topological funny-business.

For one final example of the impact of quantum mechanics on our understanding of time travel, we can return to the task of distinguishing time travel from travel to alternate worlds by examining the tradition initiated by Hugh Everett's (1957) work on quantum mechanics. Recently, philosophers have been busy clarifying and defending the philosophical underpinnings of the Everettian interpretation of quantum mechanics, and unfortunately I can only communicate the gist of the scheme, taking shortcuts and disregarding technicalities. Readers are invited to examine Jeffrey Barrett's (2011) work on this topic in order to acquaint themselves with the range of views.

In modern versions of the Everettian framework, the state of fundamental reality at any given time is the quantum state of the multiverse, which can be thought of as a field-like quantity inhabiting a very high dimensional space called 'configuration space.' As the quantum state evolves according to a deterministic equation, the many lumps in the quantum state evolve by 
undulating and continually separating into ever smaller lumps. The history of these lumps constitutes a branching structure.

What we naively think of as the present state of our physical world, according to the Everettian, exists only derivatively in virtue of a single lump in the quantum field. Each branch of the complete quantum history is called a 'world' and is supposed to correspond to a complete historical layout of macroscopic objects in space-time. Imagine the full history of how the stars and planets and rocks and animals have been arranged up until now and imagine a single way for them to develop lawfully throughout the future. That counts as one world. Then, imagine another lawful way these macroscopic entities could evolve throughout the future seemingly as a result of quantum chanciness. That counts as another world.

The continual branching of worlds implies that as time goes by, the world you inhabit is continually splitting into multiple copies, somewhat like a bacterium reproducing. Each of the daughter worlds contains a version (or successor) of you who shares the same past but evolves differently in the future. If you roll a die, for example, your world will subdivide into multiple versions, some of which contain a version of you who sees the die land on six, others of which contain a version of you who sees the die land on five, etc. The chanciness of the macroscopic world in the Everettian scheme is non-fundamental and arises in virtue of the fact that there are worlds corresponding to every possible outcome and in every such world the version of you after the roll only perceives a single outcome.

It must be emphasized that there is widespread controversy over which interpretations of quantum mechanics are tenable, and in particular a charge leveled against Everettian interpretations is that they do not account for quantum chanciness satisfactorily. But if we set aside that criticism and take for granted that the Everettian model does adequately model the kind of phenomena we observe in ordinary circumstances, we can then proceed to examine how it applies to time travel.

Deutsch and Lockwood (1994) have invoked the Everettian interpretation of quantum mechanics in order to identify a way in which a time traveler can travel to the past without being hampered by consistency constraints. Their model presupposes a fixed background space-time with a wormhole instantiating CTCs, much like the simple wormhole depicted in Fig. 1. The initial quantum state includes a potential time traveler, Sonia, who watches what comes out of the wormhole and commits herself to bilk the past by entering the wormhole if and only if she does not see her future self exit the wormhole. Classically, such a plan could not be carried out in virtue of consistency constraints, but Deutsch and Lockwood argue that there is a consistent way for Sonia to accomplish her goal. As Sonia waits by the wormhole, the possibility of a slightly older Sonia exiting the wormhole results in the following branching of worlds: In world $A$, a slightly older version of Sonia exits the wormhole followed by the slightly younger Sonia refusing to enter the wormhole, leaving two versions of Sonia to live out their lives. In world $B$, no version of Sonia exits the wormhole followed by Sonia entering the wormhole, which results in there no longer being any versions of Sonia in world $B$. In Deutsch's and Lockwood's model, the Sonia who entered the wormhole in world B exits the wormhole in world A, thereby satisfying Sonia-conservation while also allowing Sonia to satisfy her commitment to bilking by having both of her future selves fulfill their responsibilities.

This account of time travel is puzzling because on the standard way of formulating Everettian quantum mechanics, space-time and its contents are non-fundamental. One can instead use the configuration space (for the non-relativistic version at least) to serve as the fundamental container for the quantum field. Because the standard configuration space encodes for the fixed, 
three-dimensional, Euclidean character of physical space, an initial technical difficulty is to reformulate quantum mechanics to allow it to accommodate spatio-temporal curvature and non-trivial topological structure. (See Simon (1994) for a brief overview of the relevant physics and further references.) Moreover, the appeal to CTCs is motivated by the success of general relativity, a theory where space-time curvature depends on how matter is distributed. If this principle is to be wedded to an Everettian interpretation of quantum mechanics, one needs each of the distinct worlds to possess its own space-time structure, which raises a thorny technical question of how to model the effect of the many different space-time structures on the evolution of the fundamental quantum state which is supposed to evolve all worlds in tandem. Adding CTCs into the mix makes this conundrum even more puzzling. How does the appearance of a CTC in one branch of the quantum state bear on structure of the configuration space (or whatever alternative arena is employed) that contains all the quantum branches? The many unanswered questions leave it far from clear how the alleged benefits of this toy model of Everettian time travel can be sustained when one attempts to extend them into a more comprehensive theory that satisfies the broader commitments of general relativity and quantum mechanics.

Ironically, there is a sense in which the postulation of Everettian quantum mechanics renders Deutsch's and Lockwood's appeal to CTCs superfluous. After all, the non-fundamental status of space-time in Everettian models implies that in order to time travel in the Deutsch and Lockwood sense, one does not need to traverse a CTC in the fundamental arena of space-time as one would in standard models of general relativity. Presumably, one only needs the quantum state to evolve so that a world develops that is functionally similar to the way time travel is instantiated in worlds where space-time is fundamental. As it turns out, one such form of time travel is already present in the standard Everettian theory without CTCs. According to quantum mechanics generally, particles virtually always have some positive (but fantastically small!) chance of tunneling to any given remote location. Thus, there is some super-fantastically small chance of an instantaneous rearrangement of Earth's subatomic particles into just about any stable configuration. These remote possibilities are not worth bothering about in most version of quantum mechanics because of their extreme improbability, but everyone agrees that in the Everettian scheme, any future arrangement of matter that is allowed will definitely occur in some world or other. Thus, it is guaranteed that you will branch very soon into a world where all the matter on Earth has rearranged itself to match the way things were arranged some seventy million years ago. This means not only is it possible that you will appear in an environment matching the Jurassic period, and not only can you do whatever you like in such an environment without fear of consistency constraints, but astoundingly, it is determined to occur in the next few moments with no effort on your part and with no way for you to prevent it. Whether such a process constitutes bona fide time travel, I will leave for you to ponder after you find refuge from the tyrannosaurines that will soon be hunting you down.

\section{References}

Arntzenius, F. \& Maudlin, T. (2002). Time Travel and Modern Physics. In C. Callender (ed). Time, Reality and Experience (169-200). Cambridge: Cambridge University Press, 2002. Updated in Edward N. Zalta (ed.). The Stanford Encyclopedia of Philosophy (Spring 2010 Edition). 〈http://plato.stanford.edu/archives/spr2010/entries/time-travel-phys/〉.

Barrett, J. (2011). Everett's Relative-State Formulation of Quantum Mechanics. In Edward N. Zalta (ed.). The Stanford Encyclopedia of Philosophy (Spring 2011 Edition). 〈http://plato.stanford.edu/archives/spr2011/entries/qm-everett/〉.

Dainton, B. (2010). Time Travel. In Time and Space, 2nd edition. Montreal: McGill-Queen's University Press.

Deutsch, D. and Lockwood, M. (1994). The Quantum Physics of Time Travel. Scientific American 270(3), 68-74. 
Dowe, P. (2007). Constraints on Data in Worlds with Closed Timelike Curves. Philosophy of Science 74, 724-735.

Earman, J. (1995). Bangs, Crunches, Whimpers, and Shrieks: Singularities and Acausalities in Relativistic Space-times. New York: Oxford University Press.

Earman, J., Smeenk, C., and Wüttrich, C. (2009). Do the Laws of Physics Forbid the Operation of Time Machines? Synthese 169, 91-124.

Earman, J. and Wüttrich, C. (2010). Time Machines. In Edward N. Zalta (ed). The Stanford Encyclopedia of Philosophy (Winter 2010 Edition). 〈http://plato.stanford.edu/archives/win2010/entries/timemachine/l.

Everett, H. (1957). 'Relative State' Formulation of Quantum Mechanics. Reviews of Modern Physics 29, 454-462. Reprinted in (1983) J. A. Wheeler and W. H. Zurek (eds.). Quantum Theory and Measurement, 315-323.

Gödel, K. (1949). A Remark about the Relationship Between Relativity Theory and Idealistic Philosophy. In P. Schlipp (ed.). Albert Einstein: Philosopher-Scientist (557-562). La Salle, IL: Open Court.

Hawking, S. W. (1992). Chronology Protection Conjecture. Physical Review D 46(2), 603-611.

Horwich, P. Time Travel. In Asymmetries in Time. Cambridge, MA: MIT Press, 1987.

Huggett, N. (2010). Time Travel. In Everywhere and Everywhen: Adventures in Physics and Philosophy. Oxford: Oxford University Press.

Keller, S. and Nelson, M. (2001). Presentists Should Believe in Time Travel. Australasian Journal of Philosophy 79(3), 333-345.

Krasnikov, S. (2002). No Time Machines in Classical General Relativity. Classical and Quantum Gravity 19, 4109-4129.

Kutach, D. (2011). Backtracking Influence. International Studies in the Philosophy of Science 25(1), 5571.

Lewis, D. (1976). The Paradoxes of Time Travel. American Philosophical Quarterly 13, 145-152.

Lockwood, M. (2007). Time Travel. In The Labyrinth of Time: Introducing the Universe. Oxford: Oxford University Press.

Manchak, J. (2011). No No-Go: A Remark on Time Machines. Studies in History and Philosophy of Modern Physics 42, 74-76.

Meiland, J. W. (1974). A Two-Dimensional Passage Model of Time for Time Travel. Philosophical Studies $26,153-173$.

Monton, B. (2003). Presentists Can Believe In Closed Timelike Curves. Analysis 63(3), 199-202.

Savitt, S. (1994). The Replacement of Time. Australasian Journal of Philosophy 72(4), 463-474.

Sider, T. (2005). Traveling in A- and B-Time. The Monist 88, 329-335.

Simon, J. Z. (1994). The Physics of Time Travel. Physics World, 27-33.

Smeenk, C. and Wüttrich, C. (2011). Time travel and time machines. In C. Callender (ed.). The Oxford Handbook of Philosophy of Time (577-630). Oxford: Oxford University Press.

Thorne, K. (1994). Black Holes and Time Warps: Einstein's Outrageous Legacy. New York: Norton.

Wells, H. G. (1895). The Time Machine. London: Heinemann.

Yourgrau, P. (1991). The Disappearance of Time: Kurt Gödel and the Idealistic Tradition in Philosophy. Cambridge: Cambridge University Press.

\section{Further Reading}

There are numerous high-quality introductions to the philosophical issues raised by time travel. I encourage interested readers to explore the chapters on time travel in Barry Dainton's Time and Space (2010), Nick Huggett's Everywhere and Everywhen (2010), Michael Lockwood's The Labyrinth of Time (2007), and the chapter by Chris Smeenk and Christian Wüttrich in The Oxford Handbook of Philosophy of Time (2011).

\section{DOUGLAS KUTACH, 2013}

Keywords: time travel, CTC, closed time-like curve, grandfather paradox, presentism!and time travel, time machines, Gödel, quantum mechanics!and time travel, Everettian quantum mechanics!and time travel, consistency constraints, chronology protection conjecture, cosmic 
censorship hypothesis, determinism, personal time, external time, singularities, wormholes, bilking

Douglas Kutach's research ranges over topics in the philosophy of physics and metaphysics. He is the author of Causation and Its Basis in Fundamental Physics as well as articles on causal asymmetry, reduction, and philosophical methodology. 